\title{
Combined Video-Assisted Thoracic Surgery and Posterior Spinal Surgery for the Treatment of Dumbbell Tumor of the First Thoracic Nerve Root
}

\author{
Junichi Ohya ${ }^{1,2}$, Kota Miyoshi ${ }^{2}$, Tomoaki Kitagawa ${ }^{2}$, Yusuke Sato ${ }^{2}$, Takamitsu Maehara $^{3}$, Yoji Mikami ${ }^{2}$ \\ ${ }^{I}$ Department of Orthopaedic Surgery, Faculty of Medicine, University of Tokyo, Tokyo, Japan \\ ${ }^{2}$ Department of Orthopaedic Surgery, Yokohama Rosai Hospital, Yokohama, Japan \\ ${ }^{3}$ Department of Respiratory Surgery, Yokohama Rosai Hospital, Yokohama, Japan
}

Although several cases of a dumbbell tumor of thoracic nerve roots have been reported, reports on the surgical procedures for a dumbbell tumor of the first thoracic (T1) nerve root are rare. Surgeons should be cautious, especially when performing a surgical procedure for a dumbbell tumor of the $\mathrm{T} 1$ nerve root because the tumor is anatomically located adjacent to important organs and because the T1 nerve root composes the lower trunk of the brachial plexus with the eighth cervical nerve root. We present cases with dumbbell tumors of the T1 nerve root that were treated with combined surgical treatment to remove the tumor. We first performed videoassisted thoracic surgery (VATS) to release the organs anteriorly and then performed posterior spinal surgery in the prone position. The combined VATS and posterior spinal surgery may become a standard surgical procedure for the treatment of dumbbell tumors of the $\mathrm{T} 1$ nerve root.

Keywords: Video-assisted thoracic surgery; Spinal cord tumor; Minimally invasive surgical procedures; Brachial plexus

\section{Introduction}

Dumbbell tumors of the first thoracic (T1) nerve root are extremely rare. In a prior study, no tumors arising from the T1 nerve roots were documented among 81 schwannomas and 14 neurofibromas [1]. A patient with a dumbbell tumor of the T1 nerve presents with motor weakness of the hand, unlike other thoracic dumbbell tumors, because the $\mathrm{T} 1$ nerve root composes the lower trunk of the brachial plexus with the eighth cervical nerve root. Horner' syndrome, caused by a dumbbell tumor of the T1 nerve root, has also been reported $[2,3]$. The risk for vascular injury during tumor removal has been noted because plexus tumors, such as dumbbell tumors of the T1 nerve root, can adhere to major vessels $[4,5]$. The functional and anatomical features of the T1 nerve root require specific management in the treatment of dumbbell tumors that arise from this nerve root.

Surgical treatment, particularly video-assisted thoracic surgery (VATS), for thoracic dumbbell tumors has been extensively reported [6-8]. A cadaveric study regarding VATS for the exposure of the proximal inferior brachial plexus was previously published [9]; however, little is known about surgical treatment for dumbbell tumors of the T1 nerve using VATS. In the present report, we describe two cases of dumbbell tumors of the T1 nerve root

Received Nov 12, 2014; Revised Nov 13, 2014; Accepted Dec 1, 2014

Corresponding author: Junichi Ohya

Department of Orthopaedic Surgery, Faculty of Medicine, University of Tokyo,

7-3-1 Hongo, Bunkyo-ku, Tokyo 113-0033, Japan

Tel: +81-3-3815-5411, Fax: +81-3-1818-4082, E-mail: oyaj-ort@h.u-tokyo.ac.jp 
that were treated with a combination of VATS and posterior spinal surgery with partial costotransversectomy.

\section{Technical Note}

\section{Case 1}

An 18-year-old woman presented with a 2 month history of numbness and motor weakness in her left upper extremity. She was referred to Yokohama Rosai Hospital after she was diagnosed with a dumbbell tumor of the thoracic spine. Magnetic resonance imaging showed a dumbbell-shaped, left foraminal and paravertebral tumor at the T1-T2 level (Fig. 1). An imaging study revealed a dumbbell tumor of the left T1 nerve root (Eden type IV). Computed tomography angiography revealed a dumbbell tumor of the left T1 nerve root, which was attached to the left vertebral and subclavian arteries and extended into the thoracic cavity (Fig. 2).

The patient underwent two-staged surgery. Initially, VATS in the right lateral position was performed by a respiratory surgeon to release the anterior organs, including the subclavian artery, vertebral artery, and parietal pleura. Subsequently, the patient was placed in the prone position and received an arc incision. The tumor was identified with T2 partial costotransversectomy. There was no response to intraoperative nerve stimulation on the $\mathrm{T} 1$ nerve root. The tumor was removed successfully while sacrificing the T1 nerve root. Postoperatively, the patient showed no motor deficits. Her remaining hospital course was uneventful. During the follow-up period, her preoperative motor weakness gradually improved. Imaging showed no recurrence at the latest follow-up.

\section{Case 2}

A 41-year-old woman underwent a workplace health screening, and this revealed an abnormal shadow on the apical portion of the right lung on her chest radiograph. Motor weakness of the intrinsic muscle in her right hand was noted on physical examination. Imaging demonstrated a dumbbell tumor of the right $\mathrm{T} 1$ nerve root that extended to the foramen and paravertebral region (Fig. 3). We diagnosed the patient as having a dumbbell tumor of
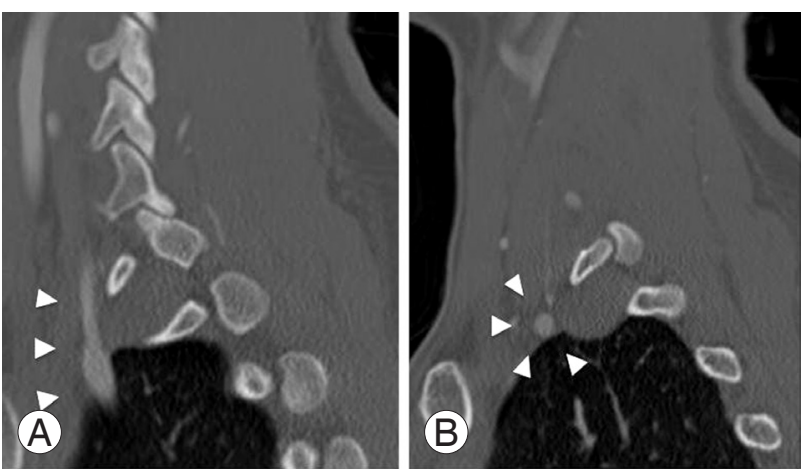

Fig. 2. Computed tomography angiography image. (A) The left vertebral artery in front of the tumor (arrowhead). (B) The left subclavian artery in front of the tumor (arrowhead).
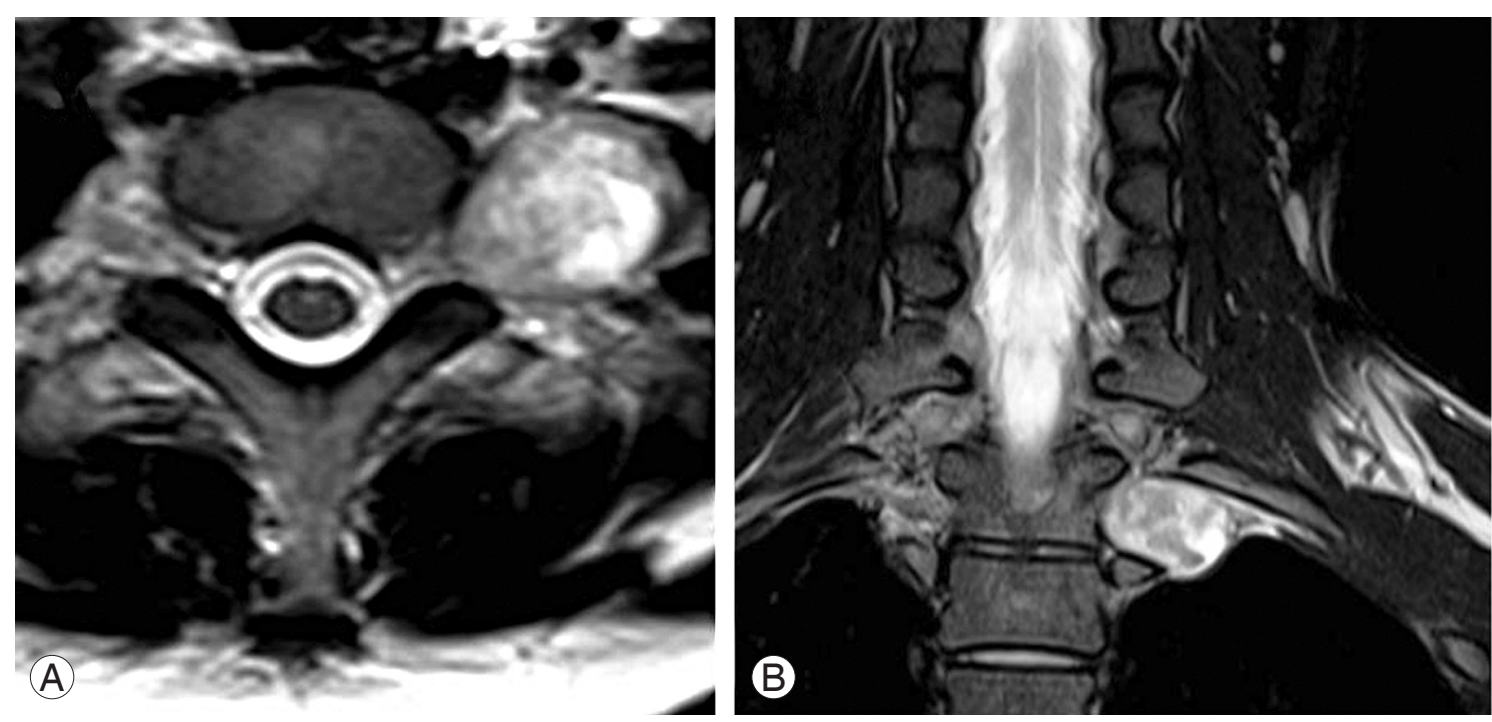

Fig. 1. T2-weighted axial and coronal magnetic resonance image (MRI). (A) Axial MRI at T1-2 level, (B) coronal MRI. 
the T1 nerve root (Eden type IV).

A combination of VATS and posterior spinal surgery was performed to remove the tumor. Anterior release using VATS in the left lateral position was performed initially (Fig. 4). During the posterior spinal surgery following VATS, a response to intraoperative nerve stimulation on the T1 nerve root distal to the tumor was confirmed. Therefore, enucleation of the tumor was performed us- ing an operating microscope to preserve intrinsic muscle function (Fig. 5). The patient had motor deficits in the flexor digitorum profundus muscle and intrinsic muscle of her right hand after surgery (grades 3 and 5, respectively), which improved slightly during the follow-up period (grades 4 and 5, respectively). Imaging showed no recurrence at the latest follow-up.
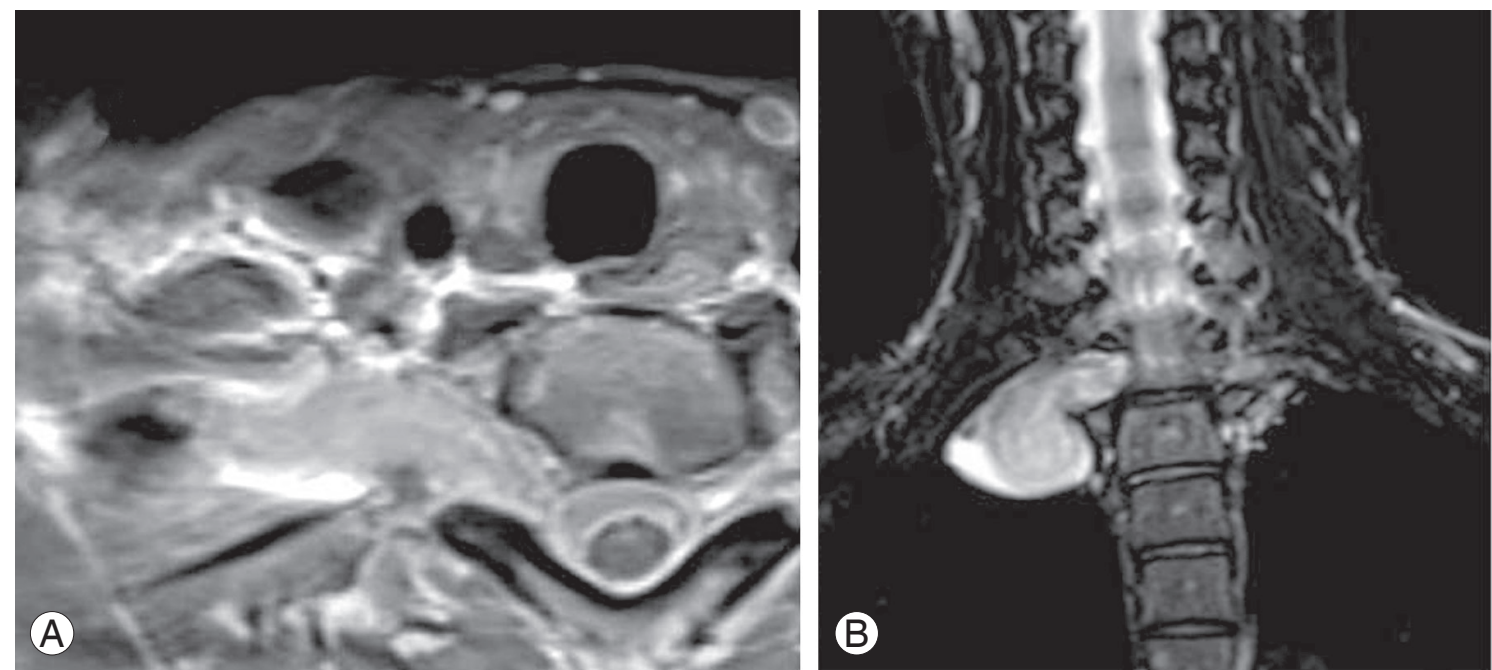

Fig. 3. T2-weighted axial and coronal magnetic resonance image (MRI). (A) Axial MRI at T1-2 level, (B) coronal MRI.
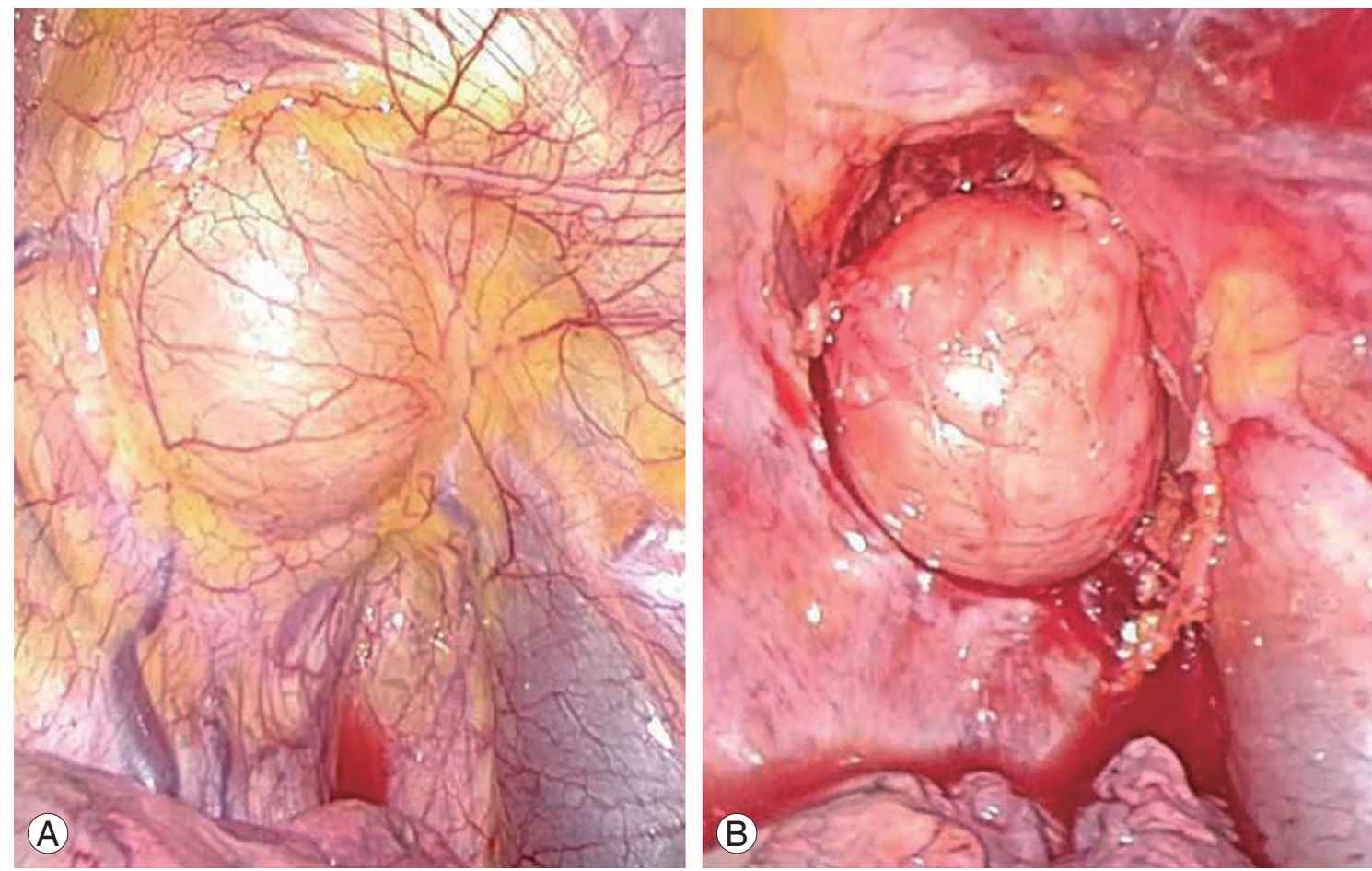

Fig. 4. (A) The tumor during video-assisted thoracic surgery. (B) The tumor after anterior release of the ambient organs. 

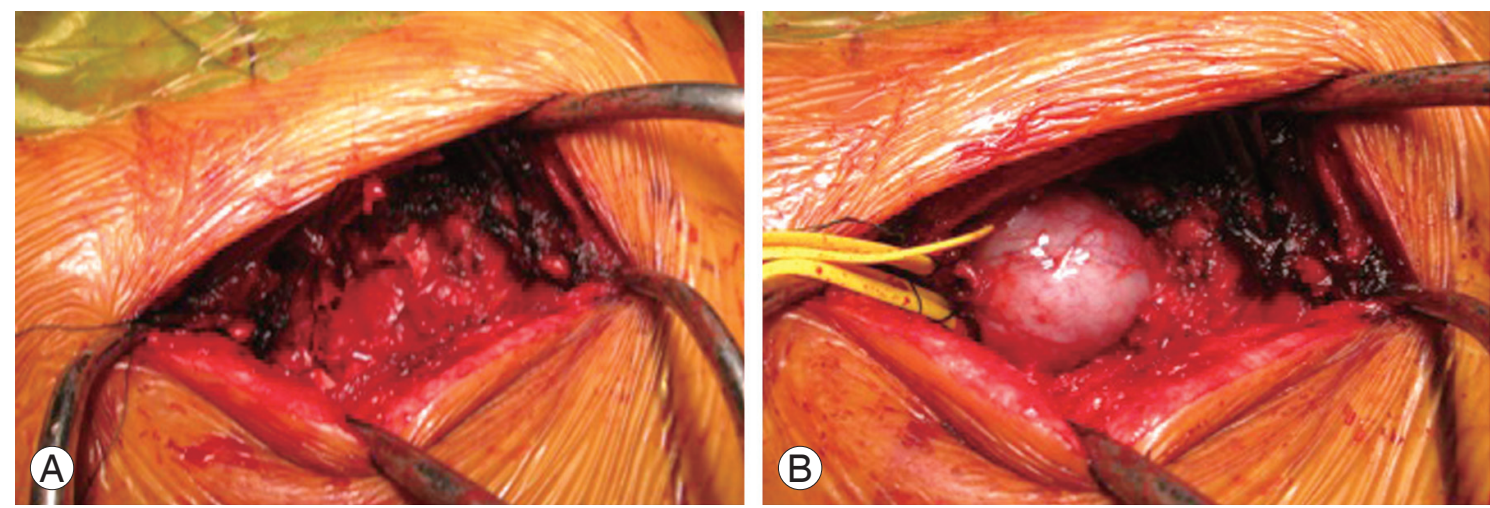

Fig. 5. Intraoperative photo in the prone position following video-assisted thoracic surgery (VATS). (A) Partial costotransversectomy was performed. (B) The preceding VATS ensured tumor mobility in the prone position. Gentle handling enabled the tumor to be moved to the surface.

\section{Pathological examination}

In both cases, pathological examinations were consistent with a diagnosis of schwannoma.

\section{Discussion}

A combined or single posterior approach has primarily been reported as the proper surgical treatment for thoracic dumbbell tumors [6-8,10]. Ando et al. [10] reported about 15 cases of thoracic dumbbell that underwent a single-stage removal of the tumor using the posterior approach. Regarding complications in their study, two cases with pleural injury and a case with a large amount of bleeding during surgery were reported. Blind posterior manipulation may lead to injury of the pleura and vessels, especially in cases of thoracic dumbbell tumors of the T1 nerve root, which is adjacent to important organs. Although the VATS technique for resection of thoracic tumors has been reported [6-8], there has been only one cadaver and case study reporting VATS for the proximal inferior brachial plexus [9]. In this prior report, the patient initially underwent a T1-T2 hemilaminectomy and facetectomy to resect the intraforaminal tumor and to transect the proximal to the dorsal root ganglion, followed by VATS; this is a sequence opposite to that used in our cases.

The cases reported here are the first to describe a combined surgical technique for treatment of dumbbell tumors of the T1 nerve root, namely VATS followed by posterior spinal surgery. One advantage of performing VATS initially is that it reduces the risk of injury to the organs adjacent to the $\mathrm{T} 1$ nerve root (the subclavian artery, vertebral artery, and thorax) during the subsequent posterior spinal surgery because they were previously released anteriorly. Because a blind procedure during posterior spinal surgery may cause massive bleeding [4,5], performing the anterior release of the organs initially through VATS could ensure that the subsequent posterior procedures can be safely performed. Second, posterior surgery is a technique familiar to spine surgeons. Surgeons are often required to perform enucleation due to encapsulation of the tumor, such as in cases of schwannoma, neurofibroma, and ganglioneuroma. Releasing the anterior organs provides a secondary advantage of obtaining tumor mobility, which can simplify the subsequent posterior surgery (Fig. 5). Although sacrificing the thoracic nerve root is generally less likely to lead to problems compared with sacrificing at the level of the cervical and lumbar nerve roots, surgeons should avoid resecting the entire T1 nerve root because of its functional importance as a component of the brachial plexus,. Therefore, intraoperative stimulation and neuromonitoring should be used to identify whether T1 nerve function is retained [11]. In cases where nerve function is retained, enucleation of the tumor using nerve-sparing techniques under a surgical microscope should be performed after separating the tumor from the adjacent functioning nerve fibers [11,12]. In such cases requiring nerve-sparing techniques, ensuring tumor mobility through VATS would help in performing microsurgery.

The T1 nerve root has anatomical and functional features that differ from those of the other thoracic nerves. In such procedurally complicated circumstances, a stan- 
dard surgical approach for dumbbell tumors of the T1 nerve root has not yet been established. However, based on the above discussion regarding the usefulness of this procedure, the combination of VATS and posterior spinal surgery could be a new standard surgical treatment for this tumor.

\section{Conflict of Interest}

No potential conflict of interest relevant to this article was reported.

\section{References}

1. Ozawa H, Kokubun S, Aizawa T, Hoshikawa T, Kawahara C. Spinal dumbbell tumors: an analysis of a series of 118 cases. J Neurosurg Spine 2007;7:58793.

2. Bosnjak R, Bacovnik U, Podnar S, Benedicic M. T1nerve root neuroma presenting with apical mass and Horner's syndrome. J Brachial Plex Peripher Nerve Inj 2007;2:7.

3. Miura J, Doita M, Miyata K, Yoshiya S, Kurosaka M, Yamamoto H. Horner's syndrome caused by a thoracic dumbbell-shaped schwannoma: sympathetic chain reconstruction after a one-stage removal of the tumor. Spine (Phila Pa 1976) 2003;28:E33-6.

4. Binder DK, Smith JS, Barbaro NM. Primary brachial plexus tumors: imaging, surgical, and pathological findings in 25 patients. Neurosurg Focus 2004;16:E11.

5. Ganju A, Roosen N, Kline DG, Tiel RL. Outcomes in a consecutive series of 111 surgically treated plexal tumors: a review of the experience at the Louisiana State University Health Sciences Center. J Neurosurg 2001;95:51-60.

6. Barrenechea IJ, Fukumoto R, Lesser JB, Ewing DR, Connery CP, Perin NI. Endoscopic resection of thoracic paravertebral and dumbbell tumors. Neurosurgery 2006;59:1195-201.

7. Citow JS, Macdonald RL, Ferguson MK. Combined laminectomy and thoracoscopic resection of a dumbbell neurofibroma: technical case report. Neurosurgery 1999;45:1263-5.

8. Konno S, Yabuki S, Kinoshita T, Kikuchi S. Combined laminectomy and thoracoscopic resection of dumbbell-type thoracic cord tumor. Spine (Phila Pa 1976) 2001;26:E130-4.

9. Morgan CJ, Lyons J, Ling BC, et al. Video-assisted thoracoscopic dissection of the brachial plexus: cadaveric study and illustrative case. Neurosurgery 2006;58:ONS-287-90.

10. Ando K, Imagama S, Ito Z, et al. Removal of thoracic dumbbell tumors through a single-stage posterior approach: its usefulness and limitations. J Orthop Sci 2013;18:380-7.

11. Kwok K, Davis B, Kliot M. Resection of a benign brachial plexus nerve sheath tumor using 8 intraoperative electrophysiological monitoring. Neurosurgery 2007;60(4 Suppl 2):316-20.

12. Siqueira MG, Martins RS, Teixeira MJ. Management of brachial plexus region tumours and tumour-like conditions: relevant diagnostic and surgical features in a consecutive series of eighteen patients. Acta Neurochir (Wien) 2009;151:1089-98. 